\title{
Prediction of the possibility of laparoscopic reduction of Petersen's hernia after gastrectomy: multicenter observational cohort study
}

\author{
Jae-Seok Min ${ }^{1}$, Jiho Park², Kyungsoo Bae³ ${ }^{3}$ Ki Young Yoon ${ }^{4}$, Tae-Han Kim5, Eun-Jung Jung ${ }^{2}$, Young-Tae Ju², \\ Chi-Young Jeong ${ }^{2}$, Ki Hyun Kim4, Young-Joon Lee², Kyung Won Seo ${ }^{4}$, Sang-Ho Jeong² \\ ${ }^{1}$ Dongnam Institute of Radiological and Medical Sciences, Cancer Center, Busan, Korea (South) \\ ${ }^{2} S$ chool of Medicine, Gyeongsang National University, Jinju, Korea (South) \\ ${ }^{3}$ Department of Radiology, School of Medicine, Gyeongsang National University, Jinju, Korea (South) \\ ${ }^{4}$ Gospel Hospital, Kosin University, Busan, Korea (South) \\ ${ }^{5}$ Department of Surgery, Gyeongsang National University, Changwon Hospital, Changwon, Korea (South)
}

Videosurgery Miniinv 2021; 16 (3): 543-551

DOI: https://doi.org/10.5114/wiitm.2021.103964

\begin{abstract}
Introduction: Petersen's hernia (PH) is a potentially fatal complication of bowel infarction that is difficult to treat by laparoscopic reduction.

Aim: To define predictive computed tomography (CT) profiles to identify PH patients who would be suitable for laparoscopic reduction by a comparative analysis between patients treated by laparoscopic and open reduction.

Material and methods: We retrospectively collected the clinical data of patients $(n=28)$ who underwent $P H$ reduction surgery after minimally invasive gastrectomy for gastric cancer in the period 2015-2018 at four training hospitals. We examined the preoperative CT scans to identify the indications for laparoscopic PH reduction.

Results: We compared the laparoscopic reduction group (laparoscopic group, $n=15$ ) and the open reduction group (open group, $n=13)$. Patients in the laparoscopic group were younger $(55.7 \pm 10.4)$ than those in the open group $(69.3 \pm 9.1)$, but there were no differences in clinical or laboratory findings. We found that there were two CT profiles with significant differences between the open and laparoscopic groups: superior mesenteric vein (SMV) narrowing and small bowel dilation. We found that small bowel dilatation was an independent factor on multivariate analysis for laparoscopic $\mathrm{PH}$ reduction.

Conclusions: We found that small bowel dilatation is the most important CT profile for identifying PH patients contraindicated for laparoscopic reduction. Despite the retrospective design of this study, these CT profiles are expected to define the scope of laparoscopic reduction in $\mathrm{PH}$ patients and to establish indications for the laparoscopic approach.
\end{abstract}

Key words: bariatric surgery, laparoscopy, hernia, gastric neoplasm, Petersen's hernia.

\section{Introduction}

Petersen's hernia (PH) is a type of long-term complication that occurs after gastrointestinal surgery, resulting in herniation between the transverse mesocolon and jejunal limb mesentery [1]. It may occur after total or distal gastrectomy (DG) with Roux-en $Y$ (RNY) reconstruction, Billroth II (B-II) reconstruction, and RNY gastrojejunal bypass. It has been reported that it occurs in approximately $2.5-6 \%$ of patients after laparoscopic RNY gastrojejunal bypass surgery performed for obesity $[2,3]$. After the small intes- 
tine herniates in Petersen's space, it progresses with intestinal necrosis or intestinal perforation. If $\mathrm{PH}$ is not diagnosed early or is misdiagnosed, it can cause sepsis in a short period of time, and the mortality rate increases dramatically. $\mathrm{PH}$ is reported to account for $0.5-5 \%$ of all cases of total small bowel obstruction, and the overall mortality rate increases by more than $50 \%$ in the case of bowel necrosis $[4,5]$. Due to this course, $\mathrm{PH}$ requires emergency surgery.

With the recent expansion of minimally invasive surgery in the surgical field, the number of reports on laparoscopic surgery in the treatment of intestinal obstruction is increasing. Laparoscopic surgery can reduce the incidence of postoperative complications compared with the open approach [6]. Compared to open adhesiolysis, the frequency of laparoscopic adhesiolysis increased from $17.6 \%$ in 2006 to $28.7 \%$ in 2013 [7]. However, some surgeons still question the application of laparoscopic surgery in treating intestinal obstruction due to the possibility of artificial intestinal damage during surgery and limited visibility. Laparoscopic surgery for the treatment of internal hernias is still reported in case reports in limited patients rather than being recognized as a standard treatment [8].

\section{Aim}

To identify predictive computed tomography (CT) profiles of patient suitability for laparoscopic reduction of $\mathrm{PH}$ by a comparative analysis between patients treated by laparoscopic and open reduction.

\section{Material and methods}

A retrospective observational study was designed and carried out according to the principles of the Declaration of Helsinki, 1989. This study was approved by the institutional review board (Approval number of corresponding author institution, GNUHIRB-201908002).

\section{Patients}

We retrospectively collected the clinical data of patients who underwent $\mathrm{PH}$ repair surgery after minimally invasive gastrectomy for gastric cancer between 2015 and 2018. Patients who underwent surgery for $\mathrm{PH}$ after a past laparoscopic or robotic gastrectomy at four hospitals were enrolled.

Six expert surgeons in laparoscopic gastrectomy have been meeting for the laparoscopic reduction of $\mathrm{PH}$ since 2015. All surgeons are specialists in gastric cancer surgery with experience in performing more than 200 laparoscopic gastrectomies. We examined preoperative CT scans and decided to perform the laparoscopic or open reduction of $\mathrm{PH}$. The recruitment of patient data was indicated after the first laparoscopic reduction by each surgeon.

Data of patients who underwent surgery from 2015 to 2018 for intestinal obstruction caused by $\mathrm{PH}$ were collected (Figure 1). The inclusion criteria of this study were as follows: 1) previous radical surgery for histologically proven primary gastric adeno-

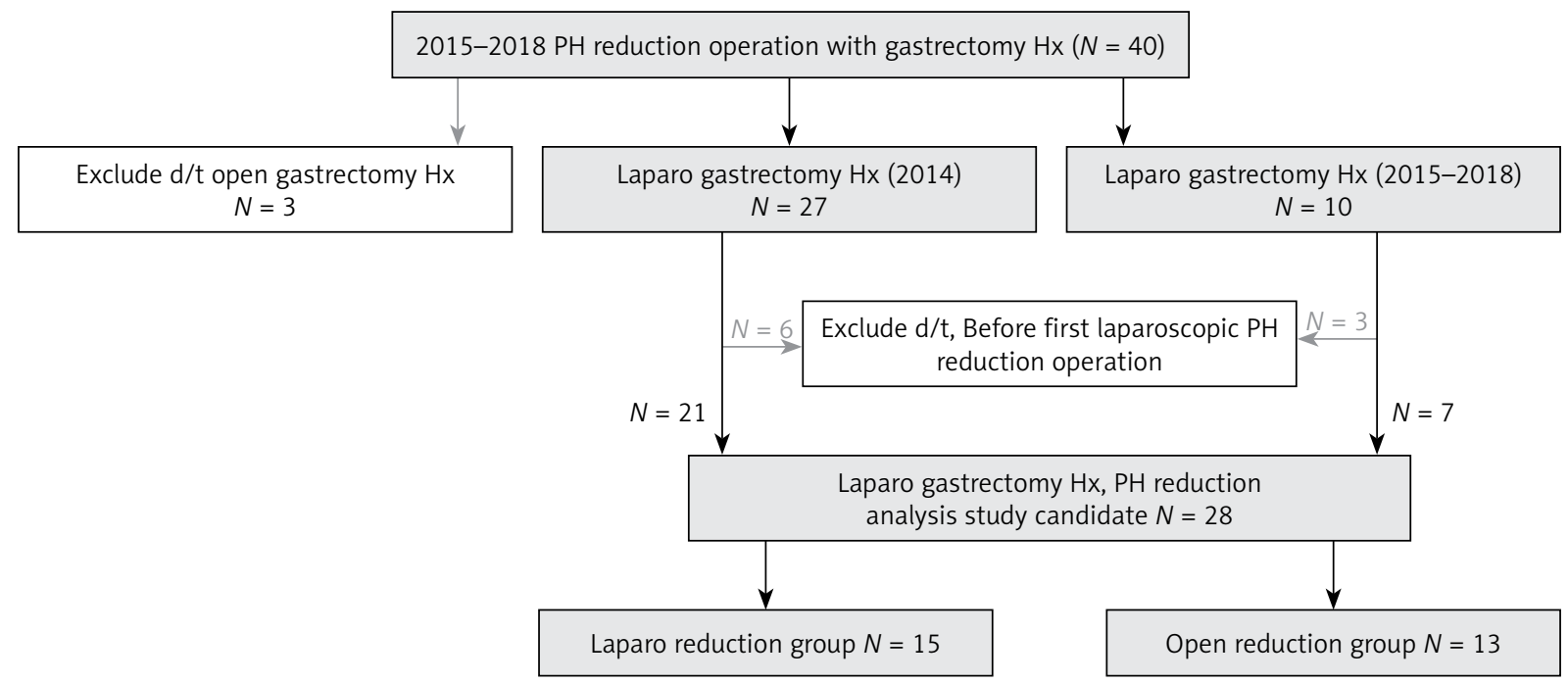

Figure 1. Patient flow diagram 
carcinoma; 2) no evidence of other distant metastases; 3) previous laparoscopic or robotic gastrectomy; and 4) reconstruction by RNY reconstruction or the gastrojejunostomy (GJ) procedure.

We excluded patients according to the following criteria: 1) preoperative patient condition of septic shock; and 2) experience of the initial operation because laparoscopic $\mathrm{PH}$ reduction is a rare procedure. There is a need to determine evidence of the possibility of laparoscopic $\mathrm{PH}$ reduction for each surgeon.

\section{Methods for CT scan}

CT scans were performed using a multidetector scanner (Brilliance iCT; Philips Healthcare, Cleveland, OH, USA). The CT scanner was set to have the following parameters: detector collimation $=64 \times$ $0.625 \mathrm{~mm}$; helical pitch $=1.173,120 \mathrm{kVp}, 120 \mathrm{effec}$ tive $\mathrm{mAs}$, section thickness/interval $=3 / 3 \mathrm{~mm}$. Abdominal surgery CT protocols included triple-phase dynamic CT. Unenhanced scans were obtained, followed by arterial-, portal-, and delayed-phase scans using a 19-s delay after the attenuation of the aorta at the thoracolumbar junction had reached $150 \mathrm{HU}$ (arterial phase), a fixed 55-s delay (portal phase) and a 275-s delay (delayed phase) after an intravenous injection of $120 \mathrm{ml}$ of iohexol (Omnipaque, GE Healthcare) administered at a rate of $3 \mathrm{ml} / \mathrm{s}$ with an autonomic injector. Abdominal pelvic CT protocols included unenhanced scans and single portal-phase scans using a fixed 70-s delay after an intravenous injection of $130 \mathrm{ml}$ of iobitridol (Xenetix 300, Guerbet) administered at a rate of $3 \mathrm{ml} / \mathrm{s}$ with an autonomic injector. Coronal reformatted images were created using the source CT dataset, with the slice thickness and reconstruction interval set to $3 \mathrm{~mm}$.

\section{Steps to analyze CT profiles for laparoscopic reduction of $\mathrm{PH}$}

1. Extraction of factors related to laparoscopic reduction after four surgeons reviewed all CT images.

2. Identification of CT characteristics through crosschecking with a radiology specialist.

3. Identification of five factors in a single-variable analysis of the laparoscopic reduction $\mathrm{CT}$ profiles.

4. Prediction of the possibility of laparoscopic reduction of $\mathrm{PH}$ using significant $\mathrm{CT}$ profiles.

\section{Definitions of CT profiles for laparoscopic reduction of $\mathrm{PH}$ (Photo 1)}

1. Whirl sign: An encircling of the small bowel loop and the SMV around the superior mesenteric artery (SMA) is observed [9]; the SMV (red arrows) is twisted toward the SMA (yellow arrows) in a clockwise or counterclockwise pattern (Photos $1 \mathrm{~A}, \mathrm{~B}$ ).

2. Narrowing of the SMV [10]: An abrupt termination or narrowing of the proximal mesenteric vein occurs (more than 50\%), with distal branch dilation; SMV collapse (red arrow in Photo 1 B) and distal branch dilation are observed (orange arrowheads in Photo $1 \mathrm{C}$ ).

3. Dilated duodenum ( $\geq 3 \mathrm{~cm}$ in diameter): The $2^{\text {nd }}$ and $3^{\text {rd }}$ duodenal portions (white arrow) are filled and dilated with fluid (Photo $1 \mathrm{C}$ ).

4. Mesenteric fat stranding [11]: Increased attenuation in the mesentery is observed compared with the adjacent normal mesentery due to mesenteric venous congestion. Photo $1 \mathrm{D}$ shows extensive mesenteric fat stranding (red arrowheads).

5. Small bowel wall thickening ( $\geq 3 \mathrm{~mm}$ in diameter) [12]: Photo 1 E shows small bowel wall thickening (white arrowheads) and mesenteric fat stranding (red arrowheads).

6. Small bowel dilation (defined as diameter $\geq 3 \mathrm{~cm}$ ) [6]: Photo $1 \mathrm{~F}$ shows small bowel dilation (white arrows), and a mean number of small bowel dilations $>3 \mathrm{~cm}$ was detected (white arrow) at the level of the anterior superior iliac spine (ASIS).

7. Intraperitoneal fluid: Fluid collection is noted in the abdominal or pelvic cavity (black arrow, Photo 1 F) [6].

\section{Operations}

\section{Indications for laparoscopic reduction}

The patients with stable vital signs with no evidence of bowel perforation or necrosis underwent emergent laparoscopic reduction surgery. A surgeon decided whether it would be possible to create a laparoscopic field due to mild to moderate abdominal distension.

\section{Laparoscopic reduction}

The first trocar was inserted by an open method with a new incision at the level of the lower abdomen. Commonly, three or four trocars were used for laparoscopic reduction. The intra-abdominal reduc- 

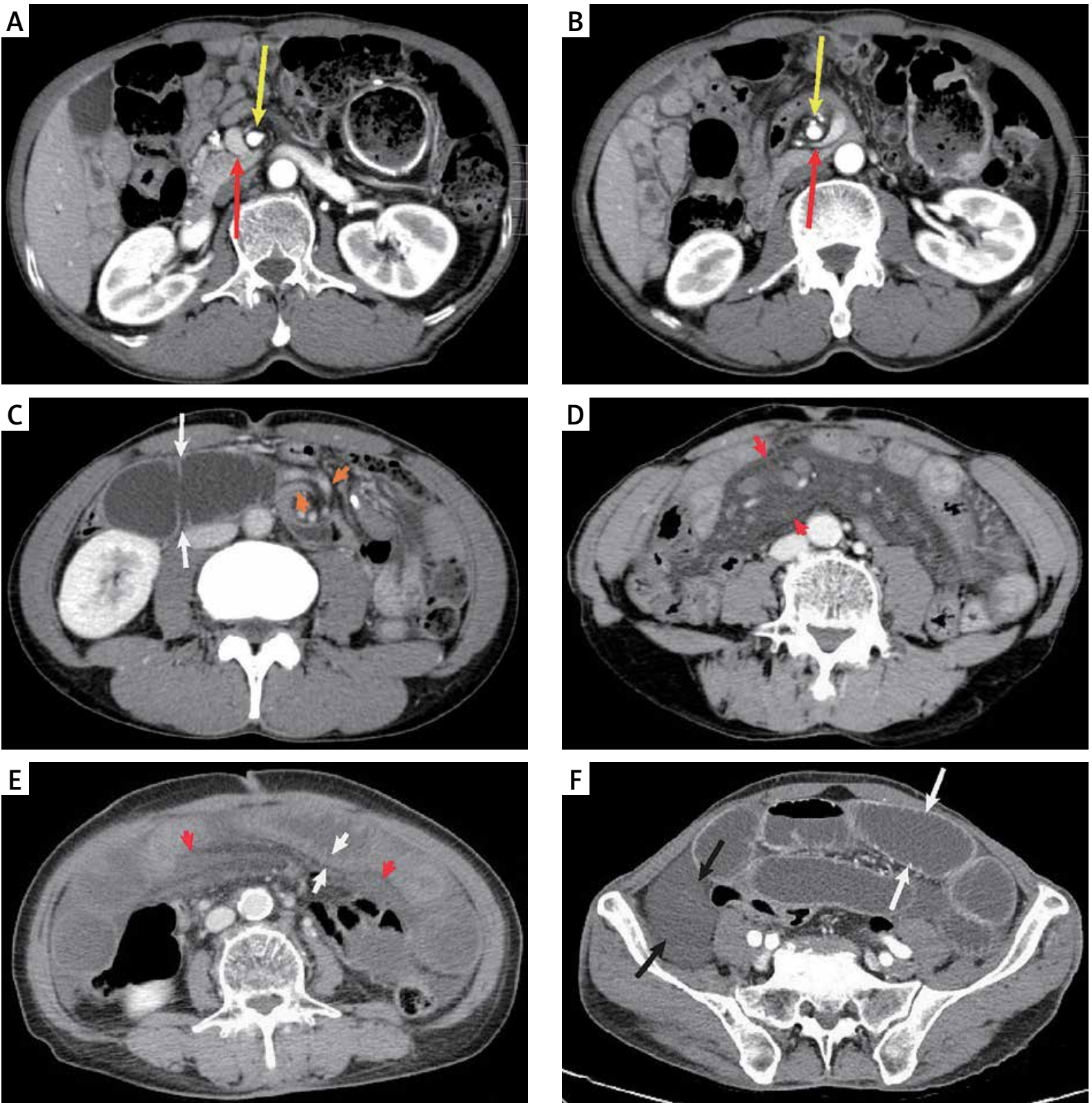

Photo 1. Characteristic CT profiles of Petersen's hernia. The proximal SMA (yellow arrow) and SMV (red arrow) were patent (A), and the distal SMA was patent, but the distal SMV (red arrow) was collapsed, as indicated by whirled mesentery (B). After whirling, the second and third duodenal portions (white arrow) were dilated and distended, the distal SMV branches were dilated (orange arrowhead) (C), and mesenteric fat stranding developed (red arrowhead, D). After SMV narrowing, the small bowel wall was thickened (white arrow), with mesenteric fat stranding (red arrowhead) in the early phase (E). In the late phase, we found small bowel dilation (white arrow) and intraperitoneal fluid (F)

tion process was the same as in the open method with laparoscopic closure of the herniated Petersen's space with nonabsorbable sutures at the final step. Careful handling of the herniated small bowel was essential because of the high possibility of bowel injury during laparoscopic reduction. If it became difficult to implement laparoscopy, for example, if severe intra-abdominal adhesions interfered with creating a pneumoperitoneum, if the laparoscopic field of view was insufficient due to excessive obstructed bowel dilation, or if the bowel or mesentery was fragile due to severe edema or bowel necrosis, 
we conducted conversion to open reduction immediately.

\section{Statistical analysis}

We used SPSS Statistics version 24 (IBM SPSS, Inc., Chicago, IL, USA) for statistical analysis. Continuous data were compared using Student's $t$-test and are presented as the means \pm standard deviations, and noncontinuous variables were assessed with the $\chi^{2}$ test. In all analyses, $p$-values less than 0.05 were considered statistically significant. A binary logistic regression model was used for univariate and multivariate analyses.

\section{Results}

\section{Patient demographics}

We found $40 \mathrm{PH}$ patients with a history of gastrectomy who underwent reduction surgery between 2015 and 2018. Figure 1 shows the patient flow diagram. We excluded 3 patients due to a history of open gastrectomy. Among 37 patients, 27 underwent gastrectomy before 2015, and 10 underwent gastrectomy between 2015 and 2018. The incidence of PH was $0.4 \%(10 / 2417)$ among gastrectomy patients from 2015 to $2018(n=2417)$, and the incidence of PH among GJ or esophagojejunostomy (EJ) patients was $1.3 \%$ (10/758).

Thirty-seven patients with a history of laparoscopic gastrectomy developed $\mathrm{PH}$ and underwent surgery, and 9 patients were excluded because the surgeon had no experience with laparoscopic hernia reduction. Therefore, there were $28 \mathrm{PH}$ patients with a history of laparoscopic gastrectomy eligible for analysis. We compared the laparoscopic group $(n=15)$ and the open reduction groups $(n=13$, 3 open conversion +10 open cases).

Table I shows the demographics of the patients. The average patient age was $62.0 \pm 11.8$ years. The male-to-female ratio was approximately $6: 1$. Analysis of the TNM stages revealed stage I, stage II and unknown stage in $64.3 \%(n=18), 21.4 \%(n=6)$ and $14.2 \%(n=4)$ of patients, respectively. Among the types of previous operations, DG with B-II was the most common procedure (39.3\%, $n=11)$, followed by total gastrectomy with RNY EJ (RNY-EJ, 25\%, $n=2$ ), DG with RNY GJ (RNY-GJ, 7.1\%, $n=2$ ), DG with uncut RNY-GJ $(12.0 \%, n=9)$, proximal gastrectomy with double tract reconstruction $(7.1 \%, n=2)$, and 2 un- known procedures due to the previous operations being performed at different hospitals. The mean period between the previous operation and the hernia reduction operation was $26.2 \pm 27.4$ months.

Regarding the operative information, there was 1 case of previous Petersen's space closure, 20 cases of no repair and 7 cases of unknown repair due to the previous operation being performed at a different hospital. The time between abdominal pain and the hernia operation was $20.7 \pm 20.6 \mathrm{~h}$. Regarding the approach, laparoscopic reduction, open reduction, and open conversion were performed in $53.6 \%(n=15), 35.7 \%(n=10)$, and $10.7 \%(n=3)$ of

Table I. Patients' demographics

\begin{tabular}{|c|c|}
\hline Factors & Value \\
\hline Age [years] & $62.0 \pm 11.8$ \\
\hline \multicolumn{2}{|l|}{ Sex: } \\
\hline Male & $24(85.7 \%)$ \\
\hline Female & $4(14.3 \%)$ \\
\hline \multicolumn{2}{|l|}{ TNM stage of gastric cancer*: } \\
\hline । & $18(64.3 \%)$ \\
\hline ॥ & $6(21.4 \%)$ \\
\hline Unknown & $4(14.2 \%)$ \\
\hline \multicolumn{2}{|l|}{ Previous operation: } \\
\hline TG with RNY-EJ & $7(25 \%)$ \\
\hline DG with B II & $11(39.3 \%)$ \\
\hline DG with RNY-GJ & $2(7.1 \%)$ \\
\hline DG with uncut RNY-GJ & $4(14.3 \%)$ \\
\hline PG with double tract & $2(7.1 \%)$ \\
\hline Unknown & $2(7.1 \%)$ \\
\hline \multicolumn{2}{|l|}{ Petersen's space repair: } \\
\hline No & $20(71.4 \%)$ \\
\hline Yes & $1(3.6 \%)$ \\
\hline Unknown & $7(25 \%)$ \\
\hline Periods between previous operation [months] & $26.2 \pm 27.4$ \\
\hline Time from pain to hernia operation [h] & $20.7 \pm 20.6$ \\
\hline \multicolumn{2}{|l|}{ Approach method: } \\
\hline Open reduction & $10(35.7 \%)$ \\
\hline Laparoscopic reduction & $15(53.6 \%)$ \\
\hline Open conversion of laparoscopic reduction & $3(10.7 \%)$ \\
\hline Operation time [min] & $81.5 \pm 25.6$ \\
\hline Small bowel injury during reduction & $3(10.7 \%)$ \\
\hline
\end{tabular}

${ }^{*}$ AICC TNM stage $8^{\text {th }}$ edition, GIST - gastrointestinal stromal tumor, $T G$ - total gastrectomy, RNY - Roux-en Y, EJ - esophagojejunostomy, $B$ II - Billroth II, GJ - gastrojejunostomy, $P G$ - proximal gastrectomy. 
Jae-Seok Min, Jiho Park, Kyungsoo Bae, Ki Young Yoon, Tae-Han Kim, Eun-Jung Jung, Young-Tae Ju, Chi-Young Jeong, Ki Hyun Kim, Young-Joon Lee, Kyung Won Seo, Sang-Ho Jeong

patients, respectively. The causes of open conversion from laparoscopic reduction were small bowel and mesentery thickening with severe congestion $(n=1)$ and difficulty in creating a secure intra-abdominal field via pneumoperitoneum due to excessive small intestine dilation $(n=2)$. There were 3 cases of small bowel injury during reduction. The mean operation time for reduction was $81.5 \pm 25.6 \mathrm{~min}$. The mean duration of postoperative hospital stay was $10.8 \pm 7.6$ days. There were 2 cases of mortality due to sepsis with multiorgan failure.

\section{Clinical characteristics in the laparoscopic and open reduction groups}

Comparing the laparoscopic and open groups, we found that patients in the laparoscopic group (55.7

Table II. Clinicopathologic comparison between laparoscopic reduction group (Laparo group) and open reduction group (Open group)

\begin{tabular}{|c|c|c|c|}
\hline Factors & Laparo group $(n=15)$ & Open group $(n=13)$ & $P$-value \\
\hline Age [years] & $55.7 \pm 10.4$ & $69.3 \pm 9.1$ & 0.001 \\
\hline \multicolumn{4}{|l|}{ Sex: } \\
\hline Male & 13 & 11 & 1.0 \\
\hline Female & 2 & 2 & \\
\hline \multicolumn{4}{|l|}{ Initial symptom: } \\
\hline Nausea & 6 & 5 & 0.70 \\
\hline Vomiting & 14 & 12 & 1.0 \\
\hline Pain & 0 & 2 & 1.0 \\
\hline Periods between previous operation [months] & $25.4 \pm 20.2$ & $27.3 \pm 36.1$ & 0.86 \\
\hline \multicolumn{4}{|l|}{ TNM stage of gastric cancer*: } \\
\hline I & 10 & 8 & 0.22 \\
\hline II & 5 & 1 & \\
\hline Unknown & 0 & 4 & \\
\hline \multicolumn{4}{|l|}{ Previous closure of Petersen's space: } \\
\hline No & 12 & 8 & 0.33 \\
\hline Yes & 0 & 1 & \\
\hline Unknown & 3 & 4 & \\
\hline Time from pain to hernia operation [h] & $22.4 \pm 23.0$ & $18.9 \pm 18.8$ & 0.67 \\
\hline \multicolumn{4}{|l|}{ Previous operation: } \\
\hline TG with RNY EJ & 4 & 3 & 0.48 \\
\hline DG with B II & 7 & 4 & \\
\hline DG with RNY & 1 & 1 & \\
\hline DG with uncut RNY & 3 & 1 & \\
\hline PG with double tract & 0 & 2 & \\
\hline Unknown & 0 & 2 & \\
\hline Postoperative small bowel obstruction & 1 & 0 & 1.0 \\
\hline \multicolumn{4}{|l|}{ Laboratory: } \\
\hline WBC & $8,971 \pm 2,794$ & $11,565 \pm 6,049$ & 0.09 \\
\hline ESR & $16.3 \pm 13.9$ & $31.8 \pm 34.1$ & 0.23 \\
\hline CRP & $0.7 \pm 1.1$ & $6.4 \pm 11.7$ & 0.12 \\
\hline
\end{tabular}

${ }^{*}$ AJCC TNM stage 8th edition, GIST - gastrointestinal stromal tumor, TG - total gastrectomy, RNY - Roux-en Y, EJ - esophagojejunostomy, B II - Billroth II, $P G$ - proximal gastrectomy, WBC - white blood cells, ESR - erythrocyte sedimentation rate, CRP-C-reactive protein. 
\pm 10.4 years) were younger than those in the open group (69.3 \pm 9.1 years, $p=0.001$, Table II). However, there were no differences between the two groups in terms of sex, initial symptoms, time since previous operation, TNM stage, previous closure of Petersen's space, time between pain and hernia operation, operation method, or inflammatory laboratory findings (e.g., white blood cell count, erythrocyte sedimentation rate, C-reactive protein level).

\section{Comparison of CT profiles between the laparoscopic and open reduction groups}

We found that all patients in the open and laparoscopic groups showed whirling signs $(p=1.0)$ (Table III, Photos 1 A, B). All patients (100\%) in the open group and $20 \%$ patients in the laparoscopic group showed SMV narrowing ( $p=0.02$, Table III, Photos $1 \mathrm{~A}, \mathrm{~B})$. There was no difference in duodenal dilation (open/laparoscopic, 7.7\%/40\%, $p=0.90$ ) (Photo $1 \mathrm{C}$, white arrow), mesentery fat stranding (open/ laparoscopic, 69.2\%/33.3\%, $p=0.32$ ) (Photo $1 \mathrm{D}$, white arrow) or small bowel wall thickening (open/ laparoscopic, $76.9 \% / 40 \%, p=0.32$ ) (Photo $1 \mathrm{E}$, white arrow) between the two groups.
Small bowel dilation was observed in $92.3 \%$ $(12 / 13)$ of patients in the open group and $13.3 \%$ $(2 / 15)$ of patients in the laparoscopic group ( $p=$ 0.01) (Photo $1 \mathrm{~F}$, white arrow). The mean numbers of small bowel dilations (more than $3 \mathrm{~cm}$ ) were $0.1 \pm 0.3$ in the laparoscopic group and $5.3 \pm 2.2$ in the open group at the ASIS level $(p<0.001)$. There was no difference in intraperitoneal fluid (open/laparoscopic, 69.2\%/33.3\%, $p=0.29$ ) (Photo $1 \mathrm{~F}$, black arrow) between the two groups.

On univariate analysis by the binary logistic regression model, narrowing of the SMV and small bowel dilation were significant CT profiles $(p<0.05$, Table IV). On multivariate analysis, small bowel dilatation was an independent factor of the laparoscopic approach for $\mathrm{PH}$ reduction $(p=0.01)$.

\section{Discussion}

The aim of this study was to identify objective predictors of patient suitability for laparoscopic emergent surgery by a comparative analysis between $\mathrm{PH}$ patients treated by laparoscopic and open reduction. We found that among the eight $\mathrm{CT}$ profiles, small bowel dilatation was the most important

Table III. Comparison of laparoscopic reduction CT profile betweenl reduction group (Laparo group) and open reduction group (Open group)

\begin{tabular}{|lccc|}
\hline CT finding & Laparo group $(n=15, \%)$ & Open group $(n=13, \%)$ & $P$-value \\
\hline Whirl sign & $15(100 \%)$ & $13(100 \%)$ & 1.0 \\
\hline Narrowing of SMV & $3(20 \%)$ & $13(100 \%)$ & 0.02 \\
\hline Dilated duodenum & $6(40 \%)$ & $1(7.7 \%)$ & 0.90 \\
\hline Mesenteric fat stranding & $5(33.3 \%)$ & $9(69.2 \%)$ & 0.32 \\
\hline Small bowel wall thickening & $6(40 \%)$ & $10(76.9 \%)$ & 0.32 \\
\hline Small bowel dilatation $(>3 \mathrm{~cm}$, at ASIS level) & $2(13.3 \%)$ & $12(92.3 \%)$ & 0.01 \\
\hline $\begin{array}{l}\text { Mean number of dilated small bowel }(>3 \mathrm{~cm}, \\
\text { at ASIS level) }\end{array}$ & $0.1 \pm 0.3$ & $5.3 \pm 2.2$ & $<0.001$ \\
\hline Intraperitoneal fluid & $5(33.3 \%)$ & $9(69.2 \%)$ & 0.29 \\
\hline
\end{tabular}

SMV - superior mesenteric vein, ASIS - anterior superior iliac spine. P-value was calculated using $\chi^{2}$ test or Student's $t$-test.

Table IV. Multivariate analysis of significant CT profiles for patients who received laparoscopic reduction for Petersen's hernia

\begin{tabular}{|c|c|c|c|c|}
\hline \multirow[t]{2}{*}{ CT finding } & \multirow{2}{*}{$\begin{array}{c}\text { Univariate analysis } \\
P \text {-value }\end{array}$} & \multicolumn{3}{|c|}{ Multivariate analysis } \\
\hline & & $P$-value & Odds ratio & $\begin{array}{l}\text { 95\% confidence } \\
\text { interval }\end{array}$ \\
\hline Narrowing of SMV & 0.02 & 0.70 & & \\
\hline Small bowel dilatation ( $>3 \mathrm{~cm}$, at ASIS level) & 0.01 & 0.01 & 0.16 & $0.03-0.74$ \\
\hline
\end{tabular}

SMV - superior mesenteric vein, ASIS - anterior superior iliac spine. P-value was calculated using binary logistic regression model. 
CT profile for identifying PH patients contraindicated for laparoscopic reduction.

$\mathrm{PH}$ is a rare disease, and the incidence of $\mathrm{PH}$ has been reported to be $0.4-9 \%$, especially after laparoscopic RNY anastomosis [3, 13-17]. Kang et al. reported that they experienced only 27 cases of $\mathrm{PH}$ among 6474 patients who underwent gastrectomy for gastric cancer [15]. In our study, the incidence of $\mathrm{PH}$ in patients who underwent laparoscopic gastrectomy with EJ or GJ was $1.3 \%$ (10/758). We also added 18 patients who underwent laparoscopic gastrectomy before 2015 . We analyzed a total of 28 cases from 4 institutions, which is not a small number of cases compared with other reports on $\mathrm{PH}$. When deciding on the surgical approach for $\mathrm{PH}$, surgeons use clinical signs, including physical findings, vital signs, laboratory data, and CT profiles, as important objective criteria. Even when laparoscopic surgery has been selected, these clinical signs are integrated. Therefore, we conducted this analysis to examine which patients are suitable for laparoscopic surgery.

In the current study, clinical data, except for patient age, did not differ between the laparoscopic and open reduction groups. Consequently, we searched for objective indicators for the laparoscopic approach. CT profiles were thought to be very useful in determining whether to perform open or laparoscopic surgery for $\mathrm{PH}$. The hypothetical processes of PH that can be identified on CT are as follows: (1) duodenal dilation indicated by whirled mesentery; (2) SMV narrowing; (3) mesenteric fat stranding due to venous congestion; (4) small bowel wall thickening due to venous congestion; (5) small bowel dilation; (6) intraperitoneal fluid collection; and (7) strangulation of the small bowel. We investigated under which conditions laparoscopic reduction could be possible.

If $\mathrm{PH}$ occurs with symptoms, such as abdominal pain, emergent reduction surgery should be performed as soon as possible using an open or laparoscopic approach. Laparoscopic surgery has several advantages over open surgery. Laparoscopic gastrectomy for gastric cancer has shown benefits over open surgery in terms of better cosmetic outcomes, lower complication rates, faster recovery, and less pain [18]. The advantages of laparoscopic surgery for the treatment of $\mathrm{PH}$ have not been reported; however, they are expected to be similar to those of laparoscopic gastrectomy. Therefore, we believe that the laparoscopic approach can en- hance patient recovery. However, there is also a possibility of intestinal injury during the insertion of trocars or the reduction of edematous dilated intestine. In the current study, therefore, it was impossible to apply laparoscopic reduction surgery in all $\mathrm{PH}$ patients.

There are many ways to detect $\mathrm{PH}$, the most obvious of which is CT. Yamashita et al. reported the typical diagnostic $\mathrm{CT}$ profiles of $\mathrm{PH}$ to differentiate it from other internal hernias in patients who have undergone gastrectomy with RNY gastric bypass [19]. They confirmed that $\mathrm{CT}$ is useful to detect PH and found that the hooking intestine sign might also be useful for diagnosing PH on CT. Additionally, other researchers have reported that $C T$ can used to precisely diagnose internal hernia after surgery [20]. They analyzed the detailed findings from abdominal CT scans evaluated by radiologists and reported a maximum sensitivity of $85.0 \%$ and a maximum specificity of $94.8 \%$. However, it was not easy to use CT profiles as objective operative indicators of $\mathrm{PH}$ due to a lack of sufficient evidence, especially regarding mesenteric and bowel edema. Additionally, the indications for open reduction or open conversion were not only CT profiles but also signs of shock. It is not easy to extract definite parameters to indicate the approach, but we attempted to extract such parameters from CT profiles. We performed a multivariate analysis of the CT profiles, which suggested that small bowel dilatation was the most significant factor for laparoscopic reduction surgery.

There were some limitations to our study. First, this was a retrospective analysis. Second, there were a small number of $\mathrm{PH}$ reduction procedures, especially open reduction procedures. Third, there is a risk of bias due to the subjective decision of the surgeon regarding the use of a laparoscopic or an open approach. Fourth, the assessments of the CT scans were subjective, including the identification of CT characteristics. However, this is the first report of an analysis of laparoscopic reduction of $\mathrm{PH}$.

\section{Conclusions}

We found that small bowel dilatation is the most important CT profile for identifying PH patients contraindicated for laparoscopic reduction. Despite the retrospective design of this study, these $\mathrm{CT}$ profiles are expected to define the scope of laparoscopic reduction in $\mathrm{PH}$ patients and to establish indications for the laparoscopic approach. 


\section{Acknowledgments}

Jae-Seok Min and Ji-Ho Park contributed equally to this study.

\section{Conflict of interest}

The authors declare no conflict of interest.

\section{References}

1. Petersen W. Ueber darmveschlingung nach der gastro-enterostomie. Arch Klin Chir 1900; 62: 94-114.

2. Iannelli A, Facchiano E, Gugenheim J. Internal hernia after laparoscopic Roux-en-Y gastric bypass for morbid obesity. Obes Surg 2006; 16: 1265-71.

3. Bauman RW, Pirrello JR. Internal hernia at Petersen's space after laparoscopic Roux-en-Y gastric bypass: 6.2\% incidence without closure: a single surgeon series of 1047 cases. Surg Obesity Relat Dis 2009; 5: 565-70.

4. Newsom BD, Kukora JS. Congenital and acquired internal hernias: unusual causes of small bowel obstruction. Am J Surg 1986; 152: 279-85.

5. Gomes R, Rodrigues J. Spontaneous adult transmesentric hernia with bowel gangrene. Hernia 2011; 15: 343-5.

6. Karkkainen JM, Saari P, Kettunen HP, et al. Interpretation of abdominal CT findings in patients who develop acute on chronic mesenteric ischemia. J Gastrointest Surg 2016; 20: 791-802.

7. Pei KY, Asuzu D, Davis KA. Will laparoscopic lysis of adhesions become the standard of care? Evaluating trends and outcomes in laparoscopic management of small-bowel obstruction using the American College of Surgeons National Surgical Quality Improvement Project Database. Surg Endosc 2017; 31: 2180-6.

8. Sakamoto T, Kawarai Lefor A. Laparoscopic reduction and closure of an internal hernia secondary to gynecologic surgery. Case Rep Surg 2017; 2017: 5948962.

9. Fisher JK. Computed tomographic diagnosis of volvulus in in testinal malrotation. Radiology 1981; 140: 145-6.

10. Duran C, Ozturk E, Uraz S, et al. Midgut volvulus: value of multidetector computed tomography in diagnosis. Turk J Gastroenterol 2008; 19: 189-92.

11. Thornton E, Mendiratta-Lala M, Siewert B, et al. Patterns of fat stranding. AJR Am J Roentgenol 2011; 197: W1-14.

12. Zalcman M, Sy M, Donckier V, et al. Helical CT signs in the diagnosis of intestinal ischemia in small-bowel obstruction. AJR Am J Roentgenol 2000; 175: 1601-7.

13. Kojima K, Inokuchi M, Kato K, et al. Petersen's hernia after laparoscopic distal gastrectomy with Roux-en-Y reconstruction for gastric cancer. Gastric Cancer 2014; 17: 146-51.

14. Himpens J, Verbrugghe A, Cadiere GB, et al. Long-term results of laparoscopic Roux-en-Y gastric bypass: evaluation after 9 years. Obes Surg 2012; 22: 1586-93.

15. Kang KM, Cho YS, Min SH, et al. Internal hernia after gastrectomy for gastric cancer in minimally invasive surgery era. Gastric Cancer 2019; 22: 1009-15.
16. Hosoya Y, Lefor A, Ui T, et al. Internal hernia after laparoscopic gastric resection with antecolic Roux-en-Y reconstruction for gastric cancer. Surg Endosc 2011; 25: 3400-4.

17. Karcz WK, Zhou C, Daoud M, et al. Modification of internal hernia classification system after laparoscopic Roux-en-Y bariatric surgery. Videosurgery Miniinv 2015; 10: 197-204.

18. Lee HJ, Hyung WJ, Yang HK, et al. Short-term outcomes of a multicenter randomized controlled trial comparing laparoscopic distal gastrectomy with $\mathrm{d} 2 \mathrm{lymphadenectomy} \mathrm{to} \mathrm{open}$ distal gastrectomy for locally advanced gastric cancer (KLASS02-RCT). Ann Surg 2019; 270: 983-91.

19. Yamashita W, Nishida K, Kawada S, et al. Hooking intestine sign: a typical diagnostic CT finding of Petersen's hernia. Japan J Radiol 2017; 35: 718-23.

20. Frokjaer JB, Jensen WN, Holt G, et al. The diagnostic performance and interrater agreement of seven CT findings in the diagnosis of internal hernia after gastric bypass operation. Abdom Radiol (NY) 2018; 43: 3220-6.

Received: 10.10.2020, accepted: 22.12.2020. 